\title{
Molecular Approaches for Understanding of the Uterine Malignant Mesenchymal Tumours
}

\author{
Takuma Hayashi ${ }^{1,7, ~ *, ~ A k i k o ~ H o r i u c h i ~}{ }^{2}$, Kenji Sano ${ }^{3}$, Nobuo Yaegashi ${ }^{4}$, Hiroyuki Aburatani ${ }^{5}$, \\ Ikuo Konishi ${ }^{6}$ \\ ${ }^{1}$ Department of Immunology and Infectious Disease, Shinshu University School of Medicine, Nagano, Japan \\ ${ }^{2}$ Horiuchi Ladies Clinic, Nagano, Japan \\ ${ }^{3}$ Department of Laboratory Medicine, Shinshu University Hospital, Nagano, Japan \\ ${ }^{4}$ Department of Obstetrics and Gynecology, Tohoku University Graduate School of Medicine, Miyagi, Japan \\ ${ }^{5}$ The Cancer System Laboratory, Research Center for Advanced Science and Technology, the University of Tokyo, Tokyo, Japan \\ ${ }^{6}$ Department of Obstetrics and Gynecology, Kyoto University Graduate School of Medicine, Kyoto, Japan \\ ${ }^{7}$ Promoting Business using Advanced Technology, Japan Science and Technology Agency (JST), Tokyo, Japan
}

Email address:

yoyoyo224@hotmail.com (T. Hayashi)

\section{To cite this article:}

Takuma Hayashi, Akiko Horiuchi, Kenji Sano, Nobuo Yaegashi, Hiroyuki Aburatani, Ikuo Konishi. Molecular Approaches for Understanding of the Uterine Malignant Mesenchymal Tumours. American Journal of Bioscience and Bioengineering. Vol. 3, No. 5, 2015 , pp. 47-49.

doi: 10.11648/j.bio.20150305.14

\begin{abstract}
Sarcomas are neoplastic malignancies that typically arise in tissues of mesenchymal origin. The identification of novel molecular mechanisms leading to mesenchymal transformation and the establishment of new therapies and biomarker has been hampered by several critical factors. First, this type of malignant tumour is rarely observed in the clinic with fewer than 15, 000 newly cases diagnosed each year in the United States. Another complicating factor is that sarcomas are extremely heterogeneous as they arise in a multitude of tissues from many different cell lineages. The scarcity of clinical materials coupled with its inherent heterogeneity creates a challenging experimental environment for clinicians and scientists. Faced with these challenges, there has been extremely limited advancement in treatment options available to patients as compared to other malignant tumours. In order to glean insight into the pathobiology of sarcomas, scientists are now using mouse models whose genomes have been specifically tailored to carry gene deletions, gene amplifications, and somatic mutations commonly observed in human sarcomas. The use of these model organisms has been successful in increasing our knowledge and understanding of how alterations in relevant oncogenic, tumour suppressive, and signaling pathways directly impact sarcomagenesis. It is the goal of many in the biological community that the use of these mouse models will serve as powerful in vivo tools to further our understanding of sarcomagenesis and potentially identify new biomarker and therapeutic strategies.
\end{abstract}

Keywords: Mesenchymal Tumour, Leimyosarcoma, PSMB9, TUMOUR PROTEIN 53 (TP53), RETINOBLASTOMA (RB)

\section{Introduction}

Sarcomas are a rare malignant tumour with less than 15 , 000 new cases diagnosed each year in the United States. Though rare, sarcomas are highly debilitating malignancies as they are often associated with significant morbidity and mortality. Sarcomas are biologically very heterogeneous as evidenced by the fact that these tumours arise from a plethora of different tissues and cell types. They are classically defined by their tissue of origin and are additionally stratified by their histopathology or patient's age at diagnosis [1]. While these classifications have proven useful, modern pathobiological and clinical techniques have the ability to further stratify sarcomas based on their genetic profile [2]. Cytogenetic and karyotype analyses have revealed two divergent genetic profiles in sarcomas. The first and most simple genetic profile is the observation of translocation events in sarcomas with an otherwise normal diploid karyotype. On the other hand, most sarcomas display a more complex genetic phenotype, suggesting genomic instability plays an important role in many sarcomas.

Proteasome beta subunit (PSMB) $9 / \beta 1 \mathrm{i}$ is encoded in the major histocompatibility complex (MHC) class region of the $20 \mathrm{~S}$ proteasome, which is part of the $26 \mathrm{~s}$ complex that 
degrades ubiquitin - conjugated proteins. A recent study reported that defective expression of PSMB9//1i may initiate the development of spontaneous human uterine leiomyosarcoma (Ut - LMS) [3]. As human mesenchymal tumours including Ut - LMS is resistant to chemotherapy and radiotherapy, and thus surgical intervention is virtually the only means of treatment, developing an efficient adjuvant therapy is expected to improve the prognosis of the sarcoma. The identification of a risk factor associated with the development of mesenchymal tumours would significantly contribute to the development of diagnostic biomarkers, preventive and therapeutic treatments.

\section{Research findings}

\subsection{IFN - $\gamma$ - Inducible Factor, PSMB9/B1i Correlates to Uterine Mesenchymal Transformation}

The proteasomal degradation is essential for many cellular processes, including the cell cycle, the regulation of gene expression and immunological function $[4,5,6]$. Interferon (IFN) - yinduces the expression of large numbers of responsive genes, subunits of proteasome $\beta$ - ring, i.e., proteasome beta subunit (PSMB) $/ \beta 1 \mathrm{i}$, PSMB5/ $\beta 5 \mathrm{i}$, and PSMB10 / multicatalytic endopeptidase complex - like (MECL) - 1/ $\beta 2 \mathrm{i}$ $[7,8]$. A molecular approach to studying the correlation of IFN - $\gamma$ with tumor cell growth has drawn attention. Homozygous mice deficient in PSMB9/ $\beta 1 \mathrm{i}$ show tissue - and substrate dependent abnormalities in the biological functions of the proteasome [7, 8, 9]. Uterine leiomyosarcoma (Ut - LMS) reportedly occurred in female PSMB9//1i - deficient mice at age 6 months or older, and the incidence at 14 months of age was about $40 \%[3,10]$. Histological studies of PSMB9/ $\beta 1 \mathrm{i}$ lacking uterine tumors have revealed characteristic abnormalities of Ut - LMS [3, 10]. Recent study, experiments with human and mouse uterine tissues revealed a defective PSMB9/ $\beta 1$ i expression in human Ut - LMS that was traced to the IFN - $\gamma$ pathway and the specific effect of somatic mutations in JANUS KINASE 1 (JAK1) molecule on the PSMB9/ $\beta 1 \mathrm{i}$ transcriptional activation [11]. Furthermore, analysis of several human Ut - LMS cell lines clarified the biological significance of PSMB9/ $\beta 1 \mathrm{i}$ in malignant myometrium transformation, thus implicating PSMB9/ $\beta 1 \mathrm{i}$ as an anti - tumorigenic candidate $[10,11]$.

\subsection{Tumour Suppressor and Oncogenic Pathways Involved in Sarcomagenesis}

Tumour protein 53 (TP53), tumour suppressor pathway is one of the most well characterized pathways in malignant tumours [12]. TP53 gene encodes a transcription factor required for the activation of numerous DNA damage dependent checkpoint response and apoptotic genes, and thus its activities are often ablated in many malignant tumours. In addition to loss of TP53 functions via inherited germline mutations, TP53 pathway is commonly disrupted by somatic mutations in TP53 gene during sporadic sarcomagenesis [13, 14]. However, even though TP53 gene alterations are widely regarded as having a significant impact on sarcomagenesis, many sarcomas retain wild type TP53, yet phenotypically display a loss of TP53 function. These findings suggest that changes in other components of TP53 pathway; such as amplification of Mouse double minute (MDM) 2 homolog, a negative regulator of TP53 pathway, may result in TP53 inactivation $[15,16]$. Furthermore, both mice and humans with elevated levels of MDM2 due to a high frequency single nucleotide polymorphism in the MDM2 promoter (Mdm2SNP309) are more susceptible to sarcoma formation [17]. Additionally, deletion or silencing of $\mathrm{p} 19^{\mathrm{Arf}}\left(\mathrm{P} 14^{\mathrm{ARF}}\right.$ in human), an inhibitor of the MDM2 - TP53 axis, often results in development of sarcomas. Together, these data indicate that while inactivation of the TP53 pathway is observed in the vast majority of human sarcomas, the mechanisms leading to disruption of the pathway can vary greatly.

RETINOBLASTOMA (RB) pathway represents a second major tumour suppressor pathway deregulated in many sarcomas. Individuals inheriting a germline RB mutation typically develop malignant tumours of the eye early in life. However, in addition to retinal malignant tumours, these children have a significantly higher propensity to develop sarcomas than the general population [18]. While inheritance of germline RB alterations increases sarcoma risk, there are also numerous examples of sporadic sarcomas harbouring spontaneous mutations and deletions of RB, particularly osteosarcomas and rhabdomyosarcomas [19]. Furthermore, $\mathrm{P} 16^{\mathrm{INK} 4 \mathrm{~A}}$, a negative regulator of the CDK - CYCLIN complexes that phosphorylate and activate $\mathrm{RB}$, is often deleted in sarcomas [20]. Together, these findings illustrate the importance of RB pathway in sarcomagenesis.

\section{Conclusions}

The vast differences in the cellular origins of sarcomas, the lack of availability of tumour specimens, and the heterogeneity inherent within individual tumours has impeded our ability to fully understand the biology of sarcomas. However, given the availability of numerous genetic knock outs, knock - ins, and conditional alleles coupled with the bevy of tissue - specific Cre - recombinase expressing mouse lines, we now have the ability to systematically and prospectively interrogate how individual genes and mutations impact sarcomagenesis. Going forward, tumor analysis from multiple murine derived tumor types can be compared and contrasted in order to identify critical changes in specific sarcomas. The molecular approaches have clearly demonstrated that while there are driver mutations/translocations, sarcomagenesis is, in fact, a multi - hit disease. The use of these mouse models mimicking the human disease condition leads to identify critical therapeutic approaches, which can be taken to lessen the impact of these debilitating diseases. Human Ut - LMS is refractory to chemotherapy and has a poor prognosis. The molecular biological and cytological information obtained from mouse tissues and human clinical materials will contribute remarkably to the development of preventive methods, a potential diagnostic - biomarker, and new 
therapeutic approaches against human Ut - LMS.

\section{References}

[1] Lasota J, Fanburg - Smith JC. (2007): Genetics for the diagnosis and treatment of mesenchymal tumors. Semin. Musculoskelet Radiol. 11 (3): 215 - 230.

[2] Taylor BS, Barretina J, Maki RG, Antonescu CR, Singer S, Ladanyi M. (2011): Advances in sarcoma genomics and new therapeutic targets. Nat. Rev. Cancer 11 (8): 541 - 557.

[3] Hayashi T, Faustman DL. (2002): Development of spontaneous uterine tumors in low molecular mass polypeptide - 2 knockout mice. Cancer Res. 62: $24-27$.

[4] Peters JM, Franke WW, Kleinschmidt JA. (1994): Distinct $19 \mathrm{~S}$ and $20 \mathrm{~S}$ subcomplexes of the $26 \mathrm{~S}$ proteasome and their distribution in the nucleus and the cytoplasm. J. Biol. Chem. 269: 7709 - 7718 .

[5] Lodish H, Berk A, Matsudaira P, Kaiser CA, Krieger M, Scott MP, Zipursky SL, Darnell J. (2004): "3". Mol Cell Biol (5th ed.). New York: W.H. Freeman and CO. 5: 66 - 72.

[6] Konstantinova IM, Tsimokha AS, Mittenberg AG. (2008): Role of proteasomes in cellular regulation. Intl. Rev. Cell Mol. Biol. 267: 59 - 124.

[7] Wang J, Maldonado MA. (2006): The Ubiquitin - Proteasome System and Its Role in Inflammatory and Autoimmune Diseases. Cell. Mol. Immunol. 3: 255 - 261.

[8] Van Kaer L, Ashton - Rickardt PG, Eichelberger M, Gaczynska M, Nagashima K, Rock KL, Goldberg AL, Doherty PC, Tonegawa S. (1994): Altered peptidase and viral - specifi c T cell response in LMP2 mutant mice. Immunity 1: 533 - 541.

[9] Hayashi T, Kodama S, Faustman D.: LMP2 expression and proteasome activity in NOD mice. (2000): Nature Medicine 6: 1064 - 1066.

[10] Hayashi T, Horiuchi A, Sano K, Hiraoka N, Kasai M, Ichimura T, Sudo T, Tagawa Y, Nishimura R, Ishiko O, Kanai Y, Yaegashi N, Aburatani H, Shiozawa T, Konishi I. (2011): Potential role of LMP2 as tumor - suppressor defines new targets for uterine leiomyosarcoma therapy. Sci. Rep. 1: 180.

[11] Hayashi T, Horiuchi A, Sano K, Hiraoka N, Kasai M, Ichimura T, Nagase S, Ishiko O, Kanai Y, Yaegashi N, Aburatani H, Shiozawa T, Konishi I. (2012): Potential role of LMP2 as an anti - oncogenic factor in human uterine leiomyosarcoma: morphological significance of calponin h1. FEBS Letters 586 (13): 1824 - 1831.

[12] Vogelstein B, Lane D, Levine AJ. (2000): Surfing the p53 network. Nature 408 (6810): 307 - 310.
[13] Raycroft L, Wu HY, Lozano G. (1990): Transcriptional activation by wild - type but not transforming mutants of the p53 anti - oncogene. Science 249 (4972): 1049 - 1051.

[14] Wang LL. (2005): Biology of osteogenic sarcoma. Cancer J. 11 (4): 294-305.

[15] Oliner JD, Kinzler KW, Meltzer PS, George DL, Vogelstein B. (1992): Amplification of a gene encoding a p53 - associated protein in human sarcomas. Nature 358 (6381): 80 - 83.

[16] Oliner JD, Pietenpol JA, Thiagalingam S, Gyuris J, Kinzler KW, Vogelstein B. (1993): Oncoprotein MDM2 conceals the activation domain of tumour suppressor p53. Nature 362 (6423): 857 - 860.

[17] Ito M, Barys L, O'Reilly T, Young S, Gorbatcheva B, Monahan J, Zumstein - Mecker S, Choong PF, Dickinson I, Crowe P, Hemmings C, Desai J, Thomas DM, Lisztwan J. (2011): Comprehensive Mapping of p53 Pathway Alterations Reveals an Apparent Role for Both SNP309 and MDM2 Amplification in Sarcomagenesis. Clin. Cancer Res. 17 (3): 416 - 426.

[18] Deshpande A, Hinds PW. (2006): The retinoblastoma protein in osteoblast differentiation and osteosarcoma. Curr. Mol. Med. 6 (7): 809 - 817.

[19] Toguchida J, Ishizaki K, Sasaki MS, Nakamura Y, Ikenaga M, Kato M, Sugimot M, Kotoura Y, Yamamuro T. (1989): Preferential mutation of paternally derived RB gene as the initial event in sporadic osteosarcoma. Nature 338 (6211): 156 158.

[20] Oda Y, Yamamoto H, Takahira T, Kobayashi C, Kawaguchi K, Tateishi N, Nozuka Y, Tamiya S, Tanaka K, Matsuda S, Yokoyama R, Iwamoto Y, Tsuneyoshi M. (2005): Frequent alteration of $\mathrm{p} 16$ (INK4a)/p 14 (ARF) and p53 pathways in the round cell component of myxoid/round cell liposarcoma: p53 gene alterations and reduced $\mathrm{p} 14$ (ARF) expression both correlate with poor prognosis. J. Pathol. 207 (4): 410 - 421.

\section{Biography}

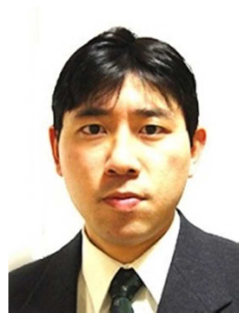

\section{Takuma Hayashi}

Dr. Hayashi is associate professor at Shinshu University School of Medicine since 2002. He has been studying the tumorigenesis of uterine leiomyosarcoma (Ut - LMS) with LMP2 - deficient mice, under the cooperation of Dr. Susumu Tonegawa (MIT). He identifies diagnostic biomarkers for Ut - LMS and focus biological characters of Ut - LMS. 